\title{
Sports medicine in the Federal Republic of Germany
}

\section{W. Hollmann, MD}

Institute für Kreislaufforschung und Sportmedizin, Cologne, FRG.

\section{Historical perspective}

The basis of sports medicine is represented by the measurement of physical performance capacity. As early as 1883, a university professor and medical doctor from Magdeburg, Speck, constructed an ergometer, and the physiologist Zuntz in Berlin treadmills. The internist Kuelbs (Cologne) trained one dog from pairs of various litters on a self-constructed treadmill and described not only the training-related muscle hypertrophy, but also the increase in size of other internal organs, such as the liver, lungs, kidneys and pancreas in 1906.

Germany is considered to be the mother country of sports medicine. In 1912, the first association of sports physicians in the world was founded in Oberhof. The term 'sports physician' was coined in Berlin in 1913. Printing of the first regularly published sports-medical journal in the world began in Berlin in 1924, and in 1928 , predominantly upon German initiative, the International Federation of Sports Medicine (FIMS) was founded in St Moritz, Switzerland.

\section{Organization of sports medicine in the FRG from 1950 to the present}

With the end of World War II in 1945, the organization of sports physicians had also collapsed. Re-establishment occurred in Hanover in 1950 under the name 'German Society of Sports Medicine'. The main task was defined as the construction of sports medicine information centres, the organization of sports medicine examinations, and the conduct of graduate courses in sports medicine.

Initially, the Diploma of the German Sports-Medical Association was enough for attainment of the title 'sports physician'. A prerequisite was a medical licence and participation in at least 4-5 weekend courses, as well as an annual sports medicine congress. To improve the quality of sports medicine, we introduced the so-called 'Additional Designation of Sports Medicine' in 1967 in conjunction with the German Society of Sports Medicine. This was approved at the German Council of Physicians in 1970 and since

Address for correspondence: Univ.-Prof. Dr Med. Dr h.c. Wildor Hollmann, Director of the Institute for Cardiology and Sports Medicine, German Sports University, 5000 Cologne, Federal Republic of Germany

(C) 1989 Butterworth \& Co (Publishers) Ltd 0306-3674/89/030142-03 \$03.00



Figure 1. German Sports University, Köln

then has been conferred by the medical boards of the various states of our Federal Republic. Participation in 240 specific hours of sports medicine instruction following the medical licence is compulsory. We object to the introduction of a specialized physician for sports medicine such as currently exists in the German Democratic Republic because we do not see it promising any practical or scientific sports-medical advantages.

The number of sports physicians totalled about 1500 at the time of re-establishment in 1950. By 1960 it had already climbed to 2800 , while the German Society of 
Sports Medicine currently comprises a total of over 9000 physicians.

International relations were restored in 1952 with the return to the International Federation of Sports Medicine (FIMS). The German Society of Sports Medicine was contracted by the International Federation of Sport Medicine in 1966 to organize the 16th World Congress for Sports Medicine in Hanover. More than 4000 physicians from over 30 nations participated. Since 1974, one member of the German Society of Sports Medicine has belonged to the International Research Committee and since 1978 also to the Executive Board of the International Federation, Hollmann acceding to the presidency of FIMS in 1986.

For many years, physicians in the German Society of Sports Medicine had been represented in all the important bodies of German sports and German medicine. This also holds true for interdisciplinary institutions of the federal medical boards, such as the scientific advisory boards, or the ministries, such as the Scientific Advisory Board of the Defence Ministry.

The focal points of sports medicine in the Federal Republic of Germany are the sports medicine centres. These did not come into existence as the result of planning on the state level, but rather purely coincidently through the initiative of individual persons. In 1950, Mellerowicz in Berlin, Reindell in Freiburg, and in Cologne Knipping with Bolt, Valentin, and Venrath were considered to be 'the men of the hour'. By 1953 they were joined by Metzner and Gadermann in Hamburg, Klaus in Münster, and Hollmann in Cologne.

At the universities, too, sports-medicine developed. As early as in 1928 the Universities of Hamburg and Leipzig introduced specific lessons for sports medicine (Knoll in Hamburg, Arnold in Leipzig). In 1956, Reindell was given a special chair for occupational physiology and sports medicine in Freiburg. This was transformed into a permanent official chair for cardiology and sports medicine in 1965.

The Institute for Cardiology and Sports Medicine of the German Sports University and the Medical University Clinic in Cologne existed in 1958 as an independent institution with its own budget and staff, founded by the author. In 1961, the Institute for Sports Medicine of the University of Münster and, 1963, the Institute for Performance Medicine in Berlin were formally opened. An official chair for cardiology and sports medicine was also created in Cologne in 1965 (Hollmann) which was connected to the administration of the Institute for Cardiology and Sports Medicine. Claus received his official chair in Münster in 1970 as did Mellerowicz in Berlin. Thus, academic development was completed in the initial centres.

A large number of sports medicine facilities rapidly came into existence in the late 1960s and especially in the 1970s. Today there are 41 professors for sports medicine at German universities. The institutions range from Institutes or Departments of Sports Medicine to individuals with teaching contracts in sports medicine.

Based on research results in preventive cardiology in 1950 and during the second half of the 1960s, sports medicine turned increasingly to the areas of motor capacity in early mobilization and active rehabilitation, especially in post-cardiac arrest states. At the same time in the health spas of the Federal Republic of

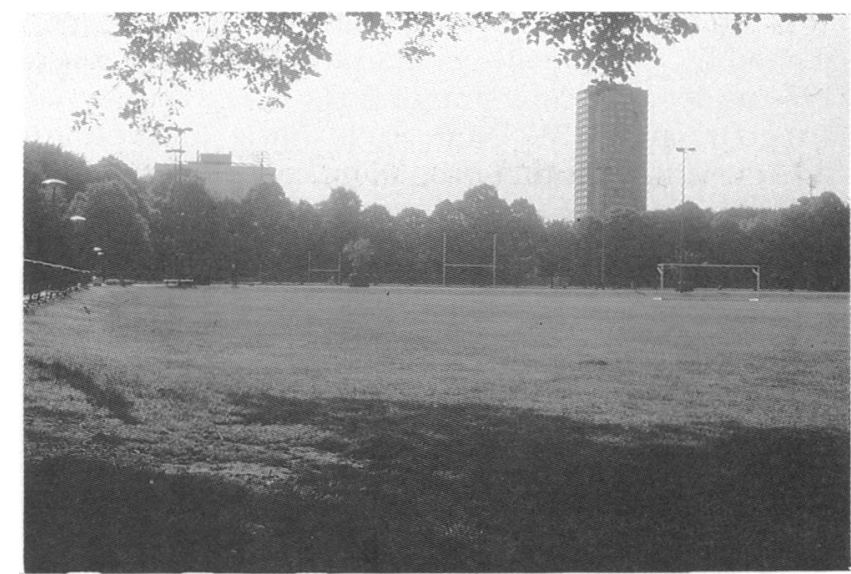

Figure 2. View to German Sports University, Köln

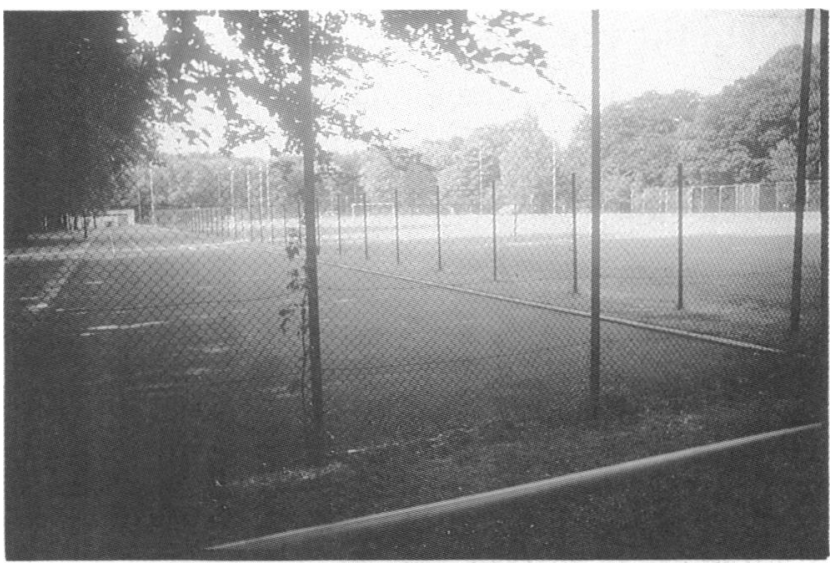

Figure 3. German Sports University, Köln; hard-court practice area

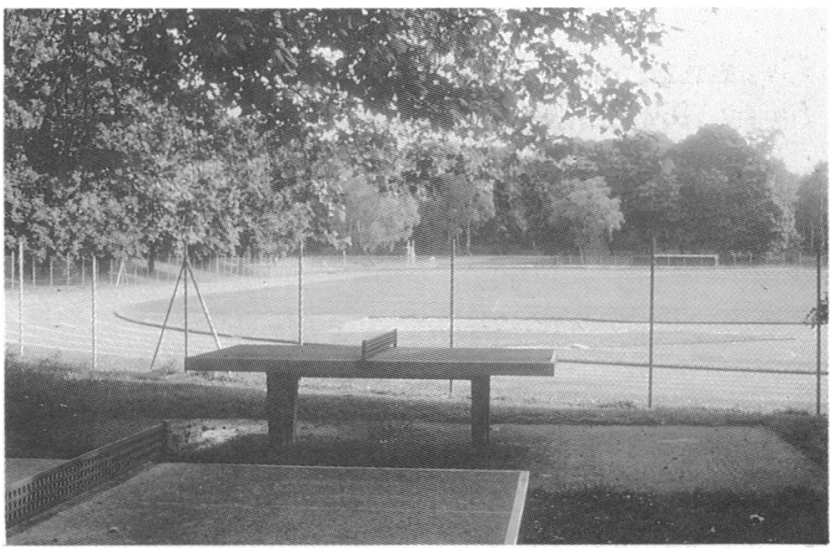

Figure 4. Training track, Köln

Germany, a revolution in priority setting took place, shifting treatment from previously passive measures to active exercise therapy. The saying was coined: "Grandpa's spa is dead". Thus, it was only logical for many of the newly-constructed rehabilitation centres to be equipped with sports-medical services.

Likewise, active outpatient measures for cardiac patients traced back to sports medicine. In 1963, Weidener in Berlin founded an outpatient coronary training group. Hartmann in Schorndorf followed with such a group in 1965, and in 1970, the extensive 'Hamburg-Model' was begun under Ilker, Donath, 
and Krasemann. The so-called 'Cologne-Model' named by the Minister of Culture, was developed in 1974 under the leadership of Rost, later together with Lagerstroem and Völker in our institute. Furthermore, the formation of other outpatient coronary groups resulted in Freiburg under Keul and in Tübingen under Jeschke. The 'Cologne-Model' was made compulsory for all communities and cities by the Ministry of Culture. Over 2000 of such heart groups currently exist in the Federal Republic of Germany under sportsmedical administration.

\section{Research results in sports medicine in the Federal Republic of Germany from 1950 to the present}

On the occasion of the founding of our institute in 1958, we defined 'sports medicine': "sports medicine contains theoretical and practical medicine which investigates the influence of exercise, training, and sport as well as the lack of exercise, on healthy and unhealthy persons of all ages to make the findings useful in prevention, therapy, and rehabilitation as well as sport itself". Accordingly, preventive and rehabilitative research and practice stand in the foreground of sports-medical interest.

There are 6 main areas of German research in sports medicine:

- Influence of exercise and training on children and youth.

- Influence of exercise and training on older and aged persons.

- Meaning of qualitatively and quantitatively different training and sport for preventive medicine (e.g. prevention of arterio-sclerosis, cardiac arrest, peripheral arterial circulatory disturbances, hypertension, diabetes mellitus, osteoporosis, depression).

- Meaning of training and sport for rehabilitation (internal, e.g. following cardiac infarction or operations as well as in orthopaedic and neurological areas).

- Influence of various diets and drugs on physical performance (e.g. proteinase-inhibitors, $\beta$-blockers, calcium-antagonists, neurotransmitters, antihypertensive drugs, hormones).

- Development of methods to optimize training and for the scientific control of training from recreational to elite sport.

In my opinion, the most important results of sports medical research in the Federal Republic of Germany since 1950 have been:

- Confirmation that the athlete's enlarged heart is a healthy one and without a danger for the athlete now or in the future.

- Endurance training of a defined quality and quantity is capable of giving a relative protection from degenerative cardio-vascular diseases. The reasons include: biochemical and biophysical adaptations in skeletal muscle with enhanced aerobic capacity of trained muscles and reduced peripheral sympa-

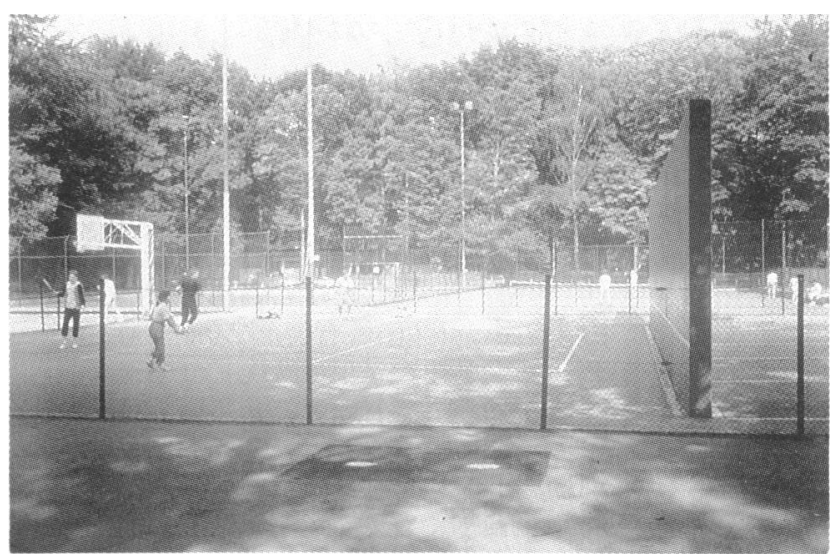

Figure 5. Training area and track, Köln

thetic drive; hence, a reduction in the oxygen demand of heart muscle; functional adaptation of the heart muscle itself with consequential lowering of oxygen demand and enhancement of electrical stability; some improvement of blood viscosity with anti-thrombotic effects; reduction of LDL-cholesterol and triglycerides, augmentation of HDLcholesterol, especially the $\mathrm{HDL}_{2}$-fraction; and reduction of catecholamines and insulin in the blood

- The possibility of reducing age-related losses in physical and mental performance capacity through different kinds of training and sport; trainability of older and aged persons with the same biochemical and biophysical adaptations in the whole body as in young people.

- Technical development of spiroergometric investigation for clinical purposes based on bicycle and crank ergometers, with blood pressure monitoring by specially developed semi-automatic apparatus combined with continuous pulse and ECG monitoring.

- First description and practical application of the principle of the aerobic-anaerobic threshold in connection with a respiratory threshold and a lactate threshold, with development of appropriate laboratory and field tests applied in patients and top athletes (1959).

- Assessment of various effects from medications during physical exercise as compared with findings during rest with consequences for therapy.

- Investigations on the influence of acute exercise on the brain blood supply and some neurotransmitters in connection with its importance for psychological and peripheral reactions.

Important aims of future research will be the continuation of the brain research in connection with exercise and investigation of the immune-system in exercise.

Around 1880, a transition from an empirically orientated medical art to a natural science-based medical technique took place. One hundred years later, a shifting of the emphasis from curative to preventive medicine began in research, teaching, and practice. Sports medicine, as a special socially oriented institution, had already taken over the administration of research and practice in this preventive area more than 30 years earlier. Its position, according to all predictions, will become even stronger in future. 